\title{
Efecto antioxidante y contenido polifenólico de Syringodium filiforme (Cymodoceaceae)
}

\author{
Kethia L. González García ${ }^{1}$, Olga Valdés Iglesias ${ }^{1}$, Abilio Laguna $^{1}$, Maylín Díaz Martínez $^{1}$ \& \\ José Antonio González Lavaut ${ }^{2}$ \\ 1. Departamento de Química. Centro de Bioproductos Marinos (CEBIMAR), Loma y 37. Nuevo Vedado, Ciudad \\ Habana, Cuba. CP. 10600; cebimar@infomed.sld.cu, kethiagg05@yahoo.es, ovaldes295@gmail.com \\ 2. Departamento de Química. Centro de Química Biomolecular. Calle 21 esquina 200. Atabey, Playa, Ciudad Habana, \\ Cuba; josealavaut@infomed.sld.cu
}

Recibido 28-VI-2010. Corregido 20-IX-2010. Aceptado 21-X-2010.

\begin{abstract}
Antioxidant effect and polyphenol content of Syringodium filiforme (Cymodoceaceae). The marine phanerogam Syringodium filiforme, known as "manatee grass", is a common species that grows in coastal areas associated to Thalassia testudinum. With the aim to describe some of its possible chemical characteristics, this study was performed with a sample of $1.2 \mathrm{~kg}$, collected in March 2009, in Guanabo beach, Havana, Cuba. The sample was dried (less than $12 \%$ humidity) and a total extract prepared; other three extracts were prepared with the use of solvents of increasing polarity. The phytochemical screening and analytical determinations of each fraction were undertaken Total polyphenol content was determined using pyrogallol as reference's standard; chlorophyll $a$ and $b$ and anthocyanin content were also quantified. Total extract and fractions antioxidant activity were evaluated by using the free radical scavenging activity assay with 1,1-Diphenyl-2-Picrylhydrazyl reactive (knowing as DPPH's method). The phytochemical screening of the different extracts detected the presence of high concentrations of flavonoids, phenols, terpenes, antocyaninns, reducing sugars and alkaloids. The total extract and methanol fraction showed significant free radical scavenging properties, while the petroleum ether fraction showed moderate activity, and the chloroform fraction and the aqueous soluble precipitate (residual salt) obtained didn't show antioxidant properties against free radicals. The results of this work confirmed the potentialities of this species for biological purposes. Rev. Biol. Trop. 59 (1): 465-472. Epub 2011 March 01.
\end{abstract}

Key words: Syringodium filiforme, phytochemistry, phenolic compounds, antioxidant activity.

La especie Syringodium filiforme Kützing in Hohenacker 1852-1962, comúnmente conocida como hierba de manatí, es una especie fanerógama perteneciente a la familia Cymodoceaceae que crece sobre fondos arenosos y sedimentos hasta $25 \mathrm{~m}$ de profundidad en el Mar del Caribe y las Antillas (Littler \& Littler 2000) en conjunto con Thalassia testudinum. Ambas especies fanerógamas son dominantes en los mares alrededor de Cuba.

Estudios recientes relacionan los efectos adversos asociados a los cambios medio ambientales sobre la fluorescencia de la clorofila $a$ y la fotosíntesis en las fanerógramas.
Según Ralph et al. (2005) factores inherentes a la elevación de la temperatura del mar, el estrés osmótico, la presencia de metales pesados $(\mathrm{Cu}$ y $\mathrm{Zn}$ ), las exposiciones a petroquímicos y la desecación por las mareas, ejercen una poderosa acción sobre especies como T. testudinum, Halophila ovalis y Zostera capricorni, y señalan su susceptibilidad y respuestas fisiológicas al estrés.

Las especies reactivas de oxígeno, producidas en los organismos debido precisamente a la incidencia de luz ultravioleta, radiaciones iónicas, reacciones químicas y procesos metabólicos causan otros daños como la 
peroxidación lipídica y proteica, daños al ADN, degeneración celular, envejecimiento y cáncer (Böhm et al. 1998, Chin-Yuang 2006), que a la vez pueden ser contrarrestadas por el efecto del consumo de compuestos como los polifenoles producidos por vegetales marinos, ya que es conocida la capacidad de capturar los radicales libres que éstos poseen.

Por otro lado, se conocen varios suplementos nutricionales elaborados a partir de extractos de plantas y organismos marinos, ricos en este tipo de compuestos de gran importancia para el mantenimiento de la salud y protección contra enfermedades relacionadas con el envejecimiento (Martínez-Flores et al. 2002, Boyd et al. 2003).

Si bien se ha demostrado la capacidad para capturar radicales libres de extractos preparados de la fanerógama marina Thalassia testudinum (Regalado et al. 2009) que convive junto a Syringodium filiforme, esta última ha sido poco estudiada. Sólo Zapata \& Mc Milan (1979), Mc Millan \& Zapata (1980) y muy recientemente Nussier et al. (2010) estudiaron esta especie desde el punto de vista químico, por lo que resulta de interés conocer si la misma posee similares cualidades farmacológicas que la especie que cohabita con ella.

\section{MATERIALES Y MÉTODOS}

Colecta y tratamiento post-cosecha: Syringodium filiforme fue recolectada el 16 de marzo de 2009 en la playa de Guanabo (2310'44” N - 82 $07^{\circ} 01^{\prime}$ ” O), La Habana, Cuba, autenticada por el Dr. Arsenio Areces, investigador del Instituto de Oceanología y depositada en la colección del Acuario Nacional de Cuba, con el número IDO 165. El material recién recolectado (1.2kg peso húmedo) fue lavado con agua potable para remover la arena y las sales, escurrido y luego secado en estufa a una temperatura de $60^{\circ} \mathrm{C}$ hasta peso constante. El contenido de humedad residual de la muestra, luego del secado en estufa, fue determinado por el método gravimétrico referido en la Farmacopea Británica (BP 2009) como referencia para los cálculos de los componentes en base seca y para la estabilidad del material de estudio.

Preparación del extracto total y fraccionamiento del mismo: Un total de $200 \mathrm{~g}$ del material vegetal seco y molido $(0.12 \mathrm{~mm})$ fueron macerados con $2000 \mathrm{ml}$ de una mezcla etanol: agua en proporción $(7: 3 ; \mathrm{v} / \mathrm{v})$; con agitación por 3 días a temperatura de $30 \pm 2^{\circ} \mathrm{C}$. Luego se procedió a la filtración mediante papel de filtro, y se realizó una segunda extracción al material vegetal con el cual se procedía de igual manera, y se unían ambos filtrados y concentrado a sequedad en un evaporador rotatorio al vacío y temperatura del baño no superior a $45^{\circ} \mathrm{C}$. Este concentrado constituyó el extracto total, el cual fue sometido a extracciones sucesivas con disolventes de menor a mayor polaridad (éter de petróleo, cloroformo y metanol).

Marcha fitoquímica: Para la determinación de las principales familias químicas presentes en la especie se evaluaron las fracciones obtenidas a partir del extracto total de diferentes polaridades, mediante reacciones de formación de color o precipitado según una modificación al método descrito por Schabra et al. 1984.

Espectroscopía UV-visible: Los espectros de UV-visible se registraron en un espectrofotómetro Shimadzu, modelo UV-1201 (Japón), con un rango de longitud de onda de 200 a $800 \mathrm{~nm}$, y se emplea celdas de cuarzo de $1 \mathrm{~cm}$ de paso óptico. Las determinaciones se realizaron a las muestras diluidas para lo cual se utiliza como blanco los respectivos disolventes de extracción (BP 2007).

Contenido de polifenoles: El contenido de polifenoles totales fue determinado por el método para la cuantificación de taninos con el reactivo de Folin-Ciocalteu (BP 2007). El extracto total y las fracciones $(200 \mu \mathrm{L})$ fueron mezclados con $800 \mu \mathrm{L}$ del reactivo ácido fosfomolíbdico-fosfotúngstico (conocido como Folin-Ciocalteu) y $200 \mu \mathrm{L} \mathrm{de} \mathrm{Na}_{2} \mathrm{Co}_{3}$ al $29 \%$. 
La mezcla fue incubada por 30 minutos a temperatura ambiente, y medida la absorbancia a $760 \mathrm{~nm}$. El contenido de polifenoles se expresó como el porcentaje relativo al peso seco del extracto y se calculó por curva de calibración usando diferentes concentraciones de pirogalol como estándar.

Determinación del contenido de clorofilas: El contenido de las clorofilas $a$ y $b$ fue determinado mediante una modificación al método propuesto en el Handbook of Food Analytical Chemistry (Waterhouse 2005) consistente en la determinación espectrofotométrica (sin el empleo de un lector ELISA de placas de 96 pocillos) a la extracción obtenida a partir $0.5 \mathrm{~g}$ del material vegetal seco y molido en $5 \mathrm{~mL}$ de metanol (Spectrosol, MERCK) con protección luminosa y por triplicado. La absorbancia fue medida a 665 y $652 \mathrm{~nm}$ y estos valores fueron utilizados para calcular la concentración de clorofila $a(\mathrm{Ca})$ y clorofila $b(\mathrm{Cb})$ basados en la ley de Lambert-Beer y los coeficientes de absorbancia tomados de la citada literatura a partir de las ecuaciones:

$$
\begin{gathered}
\mathrm{Ca}(\mu \mathrm{g} / \mathrm{ml})=16.72 \mathrm{~A}_{665}-9.16 \mathrm{~A}_{652} \\
\mathrm{Cb}(\mu \mathrm{g} / \mathrm{ml})=34.09 \mathrm{~A}_{652}-15.28 \mathrm{~A}_{665}
\end{gathered}
$$

Determinación de la concentración de antocianinas totales: Un gramo del material vegetal molido y seco se extrajo exhaustivamente, y mediante agitación constante en recipiente protegido de la luz con $50 \mathrm{~mL}$ de un mezcla metanol/ $\mathrm{HCl}$ concentrado $37 \%$ $(99: 1 v / v)$ durante $3 \mathrm{~h}$, con agitación constante. La relación peso de muestra/volumen de solvente fue $1 \mathrm{~g} / 50 \mathrm{~mL}$. El extracto obtenido se filtró, se trasvasó a un frasco volumétrico y se enrasó a $100 \mathrm{~mL}$. La absorbancia máxima a $520 \mathrm{~nm}$ se determinó y a partir de ésta se calculó la concentración de pigmentos expresado en gramos de 3,5 diglucósidos de la malvidina por cada litro de extracto, mediante la siguiente expresión:

Concentración de antocianos diglucósidos

$$
(\mathrm{g} / \mathrm{L})=\frac{\mathrm{DO}(520 \mathrm{~nm}) \times \mathrm{PM} \times \mathrm{f}}{\mathrm{E}}
$$

Donde: $\mathrm{E}^{1 \%}{ }_{1 \mathrm{~cm}}(37700 \mathrm{~L} / \mathrm{cm} . \mathrm{mol})$ es el coeficiente de extinción molar, en una cubeta de $1 \mathrm{~cm}$ de longitud, de un gramo de un compuesto con un peso molecular PM, diluido en $100 \mathrm{ml}$ de solvente. El coeficiente de extinción molar fue corregido para los diferentes disolventes. El valor del PM $691 \mathrm{~g} / \mathrm{mol}$ y f se refiere al factor de dilución. Para la determinación de los monoglucósidos se utilizó la misma expresión anterior, en cuyo caso el valor de $\mathrm{E}^{1 \%} 1 \mathrm{~cm}$ es de 28000 y un PM 529g/mol. El espectro de absorción en el rango visible a 400-580nm se determinó contra un blanco de metanol acidificado con $\mathrm{HCl}$ al $1 \%$. Todos los análisis se efectuaron por triplicado (Fuleki \& Francis 1968, Díaz et al. 1975, Fennema 1985).

\section{Evaluación de la actividad antioxidan-}

te: La actividad antioxidante se determinó a $517 \mathrm{~nm}$ para el extracto total de S. filiforme y sus fracciones añadidos a una disolución metanólica $(500 \mu \mathrm{M})$ de 1,1-difenilpicrilhidracina (DPPH) según Lai et al. 2001. El sistema de análisis incluyó la disolución DPPH y diferentes concentraciones de las muestras (entre 0.1 y 200mg), para obtener la concentración inhibitoria media $\left(\mathrm{IC}_{50}\right)$. Como blanco de compensación se utilizaron los diferentes disolventes de extracción y como control el reactivo DPPH con cada uno de los disolventes utilizados. Después de 60 minutos, se registró el descenso en la absorbancia del DPPH en presencia de la muestra el por ciento de reducción de radicales libres fue medido utilizando la siguiente ecuación:

$\%$ de reducción $=\left\{1-\frac{(\text { Abs.control-Abs muestra })}{\text { Abs. control }}\right\} \times 100$

Los resultados fueron procesados con el Programa Origin Pro 6.1 mediante análisis sigmoidal de los valores de reducción del DPPH en función de la concentración de la muestra y calcular la $\mathrm{CI}_{50}$.

\section{RESULTADOS}

El material vegetal después de ser sometido al proceso de secado, mostró una humedad 
residual igual al $10.75 \%$, valor que está por debajo del límite máximo de aceptación para la conservación de las drogas vegetales marinas secas $(<15 \%)$ según lo establece la BP (2009) para Fucus sp. y Ascophylum sp. para su protección ante hongos y levaduras.

El análisis fitoquímico realizado a los extractos a partir del material vegetal permitió identificar las familias de compuestos químicos más abundantes en la especie, y que pudieran ser responsables de su actividad biológica. Estos resultados se muestran en el Cuadro 1.

\section{CUADRO 1}

Familias de compuestos químicos encontradas en las fracciones de Syringodium filiforme

TABLE 1

Families of chemical compounds found in the fractions of Syringodium filiforme

\begin{tabular}{lcc}
\multicolumn{1}{c}{ Metabolitos } & $\begin{array}{r}\text { Tipo de } \\
\text { extracto }\end{array}$ & $\begin{array}{c}\text { Presencia de } \\
\text { metabolitos }\end{array}$ \\
Triterpenos & polar & +++ \\
Aceites esenciales & polar & - \\
Lactonas y Coumarinas & Polar y apolar & - \\
Azúcares Reductores & apolar & +++ \\
Antocianidinas & apolar & +++ \\
Compuestos fenólicos & apolar & +++ \\
Flavonoides & apolar & +++ \\
Alcaloides & apolar & ++ \\
Saponinas & apolar & ++ \\
Mucílagos & apolar & -
\end{tabular}

Como puede observarse, los compuestos fenólicos, azúcares reductores y flavonoides resultaron muy abundantes en esta especie, también fue posible detectar la presencia de antocianidinas, compuestos que se encuentran enmarcados en el grupo de los flavonoides, a los que se les atribuye actividad antioxidante (Waterhouse 2005). Otra familia de compuestos encontrados en el extracto acuoso y etanólico, fueron las saponinas y los alcaloides, aunque en menor cuantía.

Las fracciones obtenidas a partir del extracto total, analizadas por espectroscopía UV-vis, mostraron máximos de absorción característicos que están asociados a la presencia de compuestos fenólicos en general y de flavonoides (Marston \& Hostettmann 2006). Como puede observarse en el Cuadro 2, aparecen los máximos de absorción típicos de algunos núcleos de flavonoides, se observan máximos correspondientes a antocianinas (en la zona de luz visible se observa una banda entre 465-550nm y otra en la región del UV entre 270-280nm) (Andersen \& Jordheim 2006), flavonas (se observa una banda entre 304-350nm y otra entre 239$281 \mathrm{~nm}$ ) y auronas (una banda entre $370-430 \mathrm{~nm}$ y otra entre 220-270nm), además de catequinas (presencia de un máximo a 280nm) (Harborne \& Baxter 1999, Marston \& Hostettmann 2006) y se observaron máximos correspondientes a los pigmentos tipo clorofila (661-667nm).

\section{CUADRO 2}

Máximos de absorción en el espectro $U V$-visible del extracto total de $\mathrm{S}$. filiforme, de sus fracciones obtenidas y rendimientos

TABLE 2

Maximumof absorption in the spectrum $U V$-visible of total extract of $\mathrm{S}$. filiforme, their fractions and their respective yields

\begin{tabular}{lcc}
\multicolumn{1}{r}{ Tipo de extracto } & Rendimiento por fracción & $\begin{array}{c}\text { Máximos UV-vis } \\
(\mathrm{nm})\end{array}$ \\
Extracto total $($ Ext total) & $23.63 \mathrm{~g}(\mathrm{bh})$ & $664,608,534,417,312,278,240$ \\
Éter de petróleo $(\mathrm{EP})$ & $377.4 \mathrm{mg}$ & $667,533,505,411,278$ \\
Cloroformo $\left(\mathrm{CHCL}_{3}\right)$ & $12 \mathrm{mg}$ & $667,609,537,505,411,275$ \\
Metanol $(\mathrm{MeOH})$ & $1.12 \mathrm{~g}$ & $661,307,245$ \\
Sal residual & $10.54 \mathrm{~g}$ & 327 \\
Quercetina & - & 361
\end{tabular}


En el Cuadro 3 se observan los valores de concentración de polifenoles, clorofilas y antocianinas que se registran en el extracto total. Estos pigmentos fueron determinados por su posible acción como contribuyentes relativos a la actividad antioxidante del extracto. La concentración de clorofilas hallada para la especie se encuentra dentro del rango determinado por Fillit (1995) para fanerógamas marinas entre 1 y $1.8 \mu \mathrm{g} / \mathrm{ml}$.

CUADRO 3

Concentración de polifenoles totales, clorofilas y antocianinas del extracto total de $\mathrm{S}$. filiforme $(\mathrm{N}=3)$

TABLE 3

Concentration of polyphenols, chlorophylls and anthocianins of the total extract of $\mathrm{S}$. filiforme $(N=3)$

\begin{tabular}{lc}
\multicolumn{1}{c}{ Determinaciones } & Valores \\
Polifenoles b.s $(\%)$ & $25.78 \pm 0.071$ \\
Clorofilas a y b $(\mu \mathrm{g} / \mathrm{mL})$ & 1.46 \\
Relación Clorofilas a/b & 1.30 \\
Antocianinas diglicosidadas $(\mathrm{mg} / \mathrm{L})$ & 0.21 \\
Antocianinas monoglicosidadas $(\mathrm{mg} / \mathrm{L})$ & 0.22
\end{tabular}

Con referencia al contenido de polifenoles de $S$. filiforme fue superior al obtenido en el extracto crudo de Thalassia testudinum (18\% \pm 1.5$)$ (Regalado et al. 2009), así como en la fracción metanólica que confirma la abundancia de este tipo de compuestos ya detectados e identificados en la evaluación fitoquímica en los máximos de absorbancia en los espectros UV-visible y por Nussier et al. (2010) para especie similar colectada en el archipiélago de Guadalupe.

En la Fig. 1 se muestra la actividad antioxidante del extracto total en correspondencia con sus fracciones. En orden de mayor a menor, el efecto antioxidante se comportó de la manera siguiente: Extracto total (Ext total)>Fracción metanólica $(\mathrm{MeOH})>$ Fracción éter de petróleo $(\mathrm{EP})>$ Fracción clorofórmica $\left(\mathrm{CHCL}_{3}\right)>\mathrm{Sal}$ residual (Sólido Blanco).

Los valores de $\mathrm{CI}_{50}$ del extracto total y las fracciones activas se muestran en el Cuadro 4. El valor de $\mathrm{CI}_{50}$ del extracto total de S. filiforme

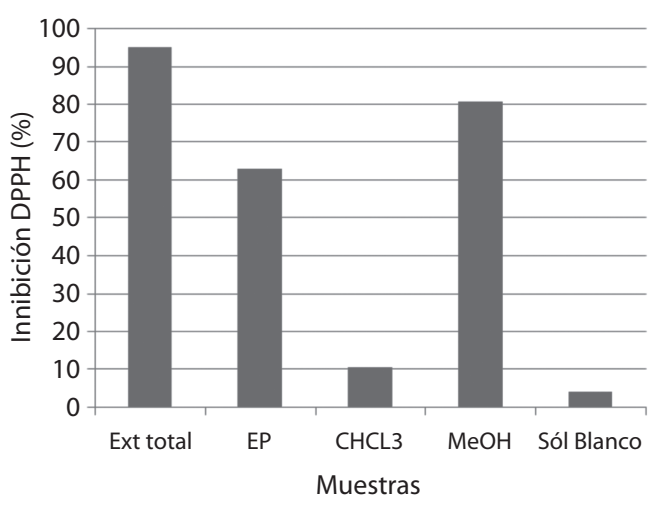

Fig. 1. Actividad antioxidante del extracto crudo de $S$. filiforme y sus fracciones medida como porcentaje de inhibición del DPPH.

Fig. 1. Antioxidant activity of the raw extract of S. filiforme and their fractions measured as inhibitory percentage of DPPH.

$(289 \mu \mathrm{g} / \mathrm{mL})$ es ligeramente superior al obtenido con el extracto total de $T$. testudinum $(158 \mu \mathrm{g} / \mathrm{mL})$. La fracción clorofórmica y la sal residual no fueron capaces de reducir el DPPH hasta un $50 \%$ lo que significa que la actividad antioxidante de ambos es baja.

\section{CUADRO 4}

Efecto inhibitorio sobre el radical DPPH y concentración de polifenoles del extracto crudo de $\mathrm{S}$. filiforme y sus fracciones activas en comparación con el extracto de T. testudinum $(N=5)$

TABLE 4

Inhibitory effect on DPPH' radical of the raw extract of $\mathrm{S}$. filiforme and their fractions and polyphenols concentration in comparison with $\mathrm{T}$. testudinum extract $(N=5)$

\begin{tabular}{lcc}
\multicolumn{1}{c}{ Muestras } & $\begin{array}{c}\mathrm{IC}_{50} \\
(\mu \mathrm{g} / \mathrm{mL})\end{array}$ & $\begin{array}{c}\text { Polifenoles } \\
(\%)\end{array}$ \\
Extracto total & 289 & $25.78 \pm 0.071$ \\
Fracción metanólica & 1663 & $11.29 \pm 1.25$ \\
Fracción éter de petróleo & 9260 & $4.50 \pm 0.46$ \\
Extracto total T. testudinum & 158 & $18 \pm 1.5$ \\
Acido Ascórbico & 2.5 &
\end{tabular}




\section{DISCUSIÓN}

Una característica beneficiosa en el análisis de los flavonoides es la presencia de anillos aromáticos dado que es un cromóforo activo en UV y de ahí la razón por la que los flavonoides son tan fácilmente detectados. Su espectro UV es particularmente informativo y proporciona una considerable información estructural que distingue el tipo de polifenol y su patrón de oxidación (Marston \& Hostettmann 2006).

Los derivados de las clorofilas pueden actuar como antioxidantes o como pro-oxidantes naturales según su concentración en la planta (Endo et al. 1985), por lo que contribuyen a la actividad antioxidante encontrada tanto en el extracto total como en las fracciones de éter de petróleo y metanólica. La bioactividad potencial de los derivados de las clorofilas en la dieta ha sido sugerida también por Ferruzzi et al. (2002) por su papel potencial como fitoquímico. Reddy et al. (2005) consideraron que la clorofila es uno de los pigmentos lipofílicos que posee una alta biodisponibilidad cuando fue ensayado junto a otros pigmentos en la inhibición del crecimiento de células tumorales, que reducen por sinergismo el impacto negativo de su actividad biológica.

Otro tanto sucede con el contenido de antocianinas, que forman parte del grupo de los flavonoides, los cuales contienen en su estructura química un número variable de grupos hidroxilo fenólicos y excelentes propiedades de quelación del hierro y otros metales de transición, lo que le confiere una fuerte actividad antioxidante (Havsteen 1983, Peres 1994, Kuskoski et al. 2004). Sus propiedades anti-radicalarias se dirigen fundamentalmente hacia los grupos hidroxilo y superóxido, especies altamente reactivas implicadas en el inicio de la cadena de peroxidación lipídica (Jovanovic et al. 1998). Los extractos de $S$. filiforme muestran la presencia de antocianinas y aunque no existe ningún valor descrito para especies marinas, es posible la contribución de estos compuestos al efecto antioxidante del extracto.
Es importante tener en cuenta a la hora de comparar la concentración inhibitoria media $\left(\mathrm{CI}_{50}\right)$ de las fanerógamas marinas: T. testudinum y $S$. filiforme, que ambas especies no fueron recolectadas en la misma zona lo que influyó significativamente en el contenido de polifenoles y en la potencia de los extractos como antioxidantes, ya que las diferencias ecológicas de las zonas de recolecta de ambas fanerógamas, puede favorecer, en mayor o menor medida, la biosíntesis de compuestos de naturaleza fenólica cuyo rol es contribuir al desarrollo y crecimiento normal de la planta y a la defensa contra infecciones y daños ecológicos (Kähkönen et al. 1999). Coincidentemente Athiperumalsami et al. (2010) encontraron que los extractos metanólicos y acuosos de la fanerógrama Halophila ovalis manifestaron una alta actividad antioxidante con valores de $\mathrm{CI}_{50}$ iguales a $78.95 \pm 1.15 \mu \mathrm{g} / \mathrm{mL}$ y $85.40 \pm 3.40 \mu \mathrm{g} /$ $\mathrm{mL}$ resultados respectivamente superiores a las fracciones y al extracto de $S$. filiforme.

Por lo tanto, al tener en cuenta estos resultados y las condiciones de trabajo empleadas, es muy posible que el efecto antioxidante correspondiente al extracto total de la especie S. filiforme se deba fundamentalmente a la presencia de compuestos de naturaleza polar (en el extracto metanólico) con una ligera contribución de los compuestos de baja polaridad (EP); lo que indica que existe un efecto sinérgico provocado por los componentes del extracto y no a un metabolito en particular.

Países como China y Japón son altamente consumidores de vegetales marinos por su alto contenido energético y sus propiedades nutricionales y antioxidantes, además de aprovechar sus propiedades farmacológicas en la preparación de formulaciones farmacéuticas, cosméticas y suplementos nutricionales (Sahoo \& Yarish 2005, Yuang 2007). De acuerdo con los resultados obtenidos, la especie $S$. filiforme constituye una posible candidata para su introducción en la dieta animal o como materia prima de un nutracéutico o de un cosmético para el consumo humano, aunque para esto se requiera de estudios toxicológicos, químicos y nutricionales complementarios. 
La especie Syringodium filiforme posee un alto contenido de polifenoles lo que justifica el efecto antioxidante detectado, particularmente para el extracto total y la fracción metanólica, resultados comparables a los encontrados por Nuissier et al. (2010) para la misma especie de Islas Guadalupe y para Thalassia testudinum de otra zona costera de Cuba. De acuerdo con estos resultados preliminares, S. filiforme pudiera ser valorada para continuar los estudios bromatológicos y toxicológicos de las especies marinas como fuente de materia prima con fines biológicos.

\section{AGRADECIMIENTOS}

Agradecemos la colaboración de José Ramón García, especialista en buceo de CEBIMAR por la recolección de la especie y posterior conservación hasta su estudio.

\section{RESUMEN}

La fanerógama marina Syringodium filiforme, conocida comúnmente como "Hierba Manatí", crece en los fondos marinos tropicales asociada con la especie Thalassia testudinum. Este estudio fue realizado con una muestra de $S$. filiforme $(1.2 \mathrm{~kg}$ peso húmedo) recolectada en la playa de Guanabo en La Habana, Cuba en Marzo 2009. La muestra fue secada hasta humedad inferior al $12 \%$ y se realizaron tres extracciones con solventes de polaridad creciente previamente al tamizaje fitoquímico y a las determinaciones analíticas. A partir del extracto total y en las fracciones se detectó la presencia de grupos químicos, fundamentalmente estructuras típicas de los flavonoides, por las absorbancias obtenidas en los espectros ultravioleta-visible. En el tamizaje fitoquímico se corroboró la existencia de altas concentraciones de flavonoides, triterpenos, polifenoles, antocianinas, azúcares reductores $\mathrm{y}$ alcaloides en los diferentes extractos obtenidos. El contenido de polifenoles totales de S. filiforme, se cuantificó mediante el uso de pirogalol como patrón de referencia; así como el contenido de clorofilas a y b, y el nivel de antocianinas. La actividad antioxidante se determinó por el método reducción del 1,1-difenil-2-picrilhidrazilo (DPPH). El extracto total y la fracción metanólica mostraron elevada actividad antioxidante en correspondencia con la concentración de polifenoles, mientras que la fracción de éter de petróleo mostró actividad moderada y la fracción clorofórmica y el precipitado remanente no muestran actividad antioxidante significativa. Estos resultados predicen las potencialidades de la especie para su uso con fines biológicos.

Palabras claves: Syringodium filiforme, fitoquímica, polifenoles, actividad antioxidante.

\section{REFERENCIAS}

Andersen, Ø.M. \& M. Jordheim. 2006. The Anthocyanins, p. 472-511. In Ø.M. Andersen \& R.K. Markham (eds.). Flavonoids: Chemistry, biochemistry and applications. CRC Press Taylor \& Francis Group, Boca de Ratón, Florida, EEUU.

Athiperumalsami, T., V.D. Rajeswari, S.H. Poorna, V. Kumar \& L.L. Jesudass. 2010. Antioxidant activity of seagrasses and seaweeds. Bot. Mar. 53: 251-257.

Böhm, H., J. Hempel, B. Raab \& A.Z. Kroke. 1998. Flavonols, flavone and anthocyanins as natural antioxidants of food and their possible role in the prevention of chronic diseases. Mnährungswiss 37: 147-63.

Boyd, S., K. Gary, C.M. Koepke, S. McAnalley, C.R. Ford, E. Horn \& B.H. McAnalley. 2003. An Open-Label Pilot Study of the Antioxidant Effect in Healthy People of Ambrotose AO ${ }^{\mathrm{TM}}$. GlycoSci. \& Nut. 4: 1-6.

British Pharmacopoeia. 2007. Vol IV (Appendix IIB) Ultraviolet and Visible Absorption Spectrophotometry. The Stationery Office, Londres, Inglaterra (también disponible en línea: www.pharmacopoeia.co.uk).

British Pharmacopoeia. 2007. Vol IV (Appendix XI M) Tannins in Herbal Drugs. The Stationery Office, Londres, Inglaterra (también disponible en línea: www. pharmacopoeia.co.uk).

British Pharmacopoeia. 2009. Volume III. Herbal Drugs and Herbal Drug Preparation Kelp The Stationery Office, Londres, Inglaterra (también disponible en línea: www.pharmacopoeia.co.uk).

Chin-Yuan, H. 2006. Antioxidant activity of extract from Polygonum aviculare L. Biol. Res. 39: 281-8.

Díaz, L., J.H. Ferrero, F. Gasque \& M. Lafuente. 1975. Estudio de los componentes antociánicos de uva de las cepas híbridas "Edo" y "Señorito". Rev. Agroq. Tecn. Alim. 15: 530-538.

Endo, Y., R. Usuki \& T. Kandena. 1985. Antioxidant effects of chlorophyll and pheophytin on the autooxidation of oils. J. Am. Oil Chem. Soc. 62: 1375-90.

Fennema, O.R. 1985. Introducción a la Ciencia de los Alimentos. Revertf, Barcelona, España. 
Ferruzzi, M., V. Bohm, P. Courtney \& S. Schwartz. 2002. Antioxidant and antimutagenic activity of dietary chlorophyll derivatives determined by radical scavenging and bacterial reverse mutagenesis assay. J. Food Sci. 67: 2589-2595.

Fillit, H. 1995: Seasonal changes in the photosynthetic capacities and pigment content of Ulva rigida in a Mediterranean Coastal Lagoon. Bot. Mar. 38: 271-280.

Fuleki, T. \& F.J. Francis.1968. Quantitative methods for anthocyanins. J. Food Sci. 33: 78-83.

Harborne, J.B. \& H. Baxter. 1999. The Handbook of Natural Flavonoids, Wiley, Nueva York, EEUU.

Havsteen, B. 1983. Flavonoids. A class of natural products of high pharmacological potency. Biochem. Pharmacol. 32: 1141-1148.

Jovanovic, S.V., S. Steenken, M.G. Simic \& Y. Hara. 1998. Antioxidant properties of flavonoids: reduction potentials and electron transfer reactions of flavonoid radicals, p. 137-161. In M. Dekker (ed.). Rice Evans C PL, Nueva York, EEUU.

Kähkönen, M.P., A.I. Hopia, H.J. Vuorela, J.P. Rauha, K. Pihlaja, T.S. Kujala \& M. Heinonen. 1999. Antioxidant activity of plant extracts containing phenolic compounds. J. Agric. Food Chem. 47: 3954-3962.

Kuskoski, E.M., A.G. Asuero, M.C. García-Parilla, A.M. Troncoso \& R. Fett. 2004. Actividad antioxidante de pigmentos antociánicos. Ciênc. Tecnol. Aliment. Campinas 24: 691-693.

Lai, L.S., S.T. Chous \& W.W. Chao. 2001. Studies on the antioxidative activities of hsian-tsao (Mesona procumbens hemsl) leaf gum. J. Agr. Food Chem. 49: 963-968.

Littler, D.S \& M.M. Littler. 2000. Caribbean reef plants. Off Shore Graphics, Florida, EEUU.

Mac Millan, C., O. Zapata \& L. Escobar. 1980. Sulphated phenolic compounds in seagrasses. Aquatic. Bot. 8: 267-278.

Marston, A. \& K. Hostettmann. 2006. Separation and Quantification of Flavonoids, p. 2-16. In Ø.M. Andersen \& R.K. Markham (eds.). Flavonoids: Chemistry, biochemistry and applications. CRC Press Taylor \& Francis Group, Boca de Ratón, Florida, EEUU.

Martínez-Flórez, S., J. González-Gallego, J.M. Culebras \& M $^{\mathrm{a}}$ J. Tuñón. 2002. Los flavonoides: propiedades y acciones antioxidantes. Nutr. Hosp. XVII. 6: 271.

Nuissier, G., B. Rezzonico \& M. Grignon-Dubois. 2010. Chicoric acid from Syringodium filiforme. Food. Chem. 120: 783-788.

Peres, W. 1994. Radicais Livres em nïveis biológicos. (ed.) Universidad Católica de Pelotas, Brasil.

Ralph, P.J. \& R. Gaderman. 2005. Rapid light curves: a powerful tool to assess photosynthetic activity. Aquat. Bot. 82: 222-237.

Reddy, M., R. Alexander-Lindo \& M. Nair. 2005. Relative inhibition of lipid peroxidation, cyclo-oxygenase, enzymes, and human tumor cell proliferation by natural foods colors. J. Agric. Food. Chem. 53: 9268-9273.

Regalado, E.L., M. Rodríguez, R. Menéndez, A.A. Concepción, C. Nogueiras, A. Laguna, A.A. Rodríguez, D.E. Williams, P. Lorenzo-Luaces, O. Valdes \& Y. Hernandez. 2009. Repair of UVB-damaged skin by the antioxidant sulphated flavone glycoside thalassiolin B isolated from the marine plant Thalassia testudinum Banks ex König. Mar. Biotechnol. 11: 74-80.

Sahoo, D. \& C. Yarish. 2005. Seaweed products find varied commercial, medical applications. Natural Products. Global Aquaculture Advocate: 44-45.

Schabra, S.C, M. Ulso \& E.N. Mshin. 1984. Phytochemical screening of Tanzanian medical plants. J. Etnopharmacol. 11: 157-159.

Waterhouse, A. 2005. Determinations of total phenolics, p. 463-470. In E. Wrolstad, T. Acree, E. Decker, M. Penner, D. Reid, S. Schwartz, C. Shoemaker, D. Smith, P. Sporns (eds.). Handbook of Food Analytical Chemistry. John Wiley \& Sons, Nueva Jersey, EEUU.

Yuang, I. 2007. Antioxidant from edible seaweed. ACS Symp. Series 956: 268-301.

Zapata, O. \& C. Mac Millan. 1979. Phenolic acids in seagrasses. Aquatic. Bot. 7: 307-317. 